MILITARY TECHNICAL COLLEGE CAIRO-EGYPT

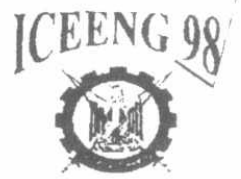

FIRST INTERNATIONAL CONF.

ON ELECTRICAL ENGINEERING

\title{
ROUNDOFF ERROR ANALYSIS OF THE SIGNED REGRESSOR ALGORITHM FOR NONSTATIONARY ADAPTIVE FILTERING
}

\author{
Eweda Eweda', Waleed M. Younis ${ }^{2}$, and Salwa H. El-Ramly ${ }^{2}$
}

\begin{abstract}
The paper is concerned with analyzing the effect of finite wordlength on the tracking performance of a signed regressor algorithm when used in the adaptive identification of a timevarying plant. Rounding quantization is assumed. Expressions of the steady state mean square error, steady state mean square weight deviation, and the corresponding optimum step sizes are derived. It is found that the mean square error, mean square weight deviation, and the optimum step sizes increase as the filter weight wordlength decreases. The effect of filter weight wordlength is found to be equivalent to an increase of the degree of nonstationarity and/or the noise of the plant. It is also found that the effect of filter weight quantization dominates the effect of input quantization. The theoretical results of the paper are validated by computer simulations.
\end{abstract}

Keywords: Adaptive Filtering, Signed Regressor Algorithm, and Roundoff Error Analysis

\section{INTRODUCTION}

Tracking analysis of the signed regressor algorithm (SRA) is available only in the infinite precision implementation of the adaptive filter[2,3]. In practical applications, however, finite wordlengths are used for the signals and adaptive filter weights. This results in quantization errors. Analysis of the effect of these errors on the algorithm performance is available only in the stationary case [5]. The present paper provides a roundoff error analysis of the tracking performance of the SRA. This analysis enables the derivation of design equations of the algorithm.

To present the notations of the paper, consider the case of adaptive identification of an unknown time varying plant $[1,11]$ depicte: $\mathrm{d}$ in figure 1 . In this figure, $a_{k}$ is the plant output at time k, $\underline{G}_{k}$ is the vector composed of the plant parameters, $\underline{X}_{k}$ is the observation vector composed of the input samples contributing to $a_{k}$, and $b_{k}$ is the plant noise. The vector $\underline{G}_{k}$ is assumed randomly time varying. The increment of $\underline{G}_{k}$ is denoted by $\underline{D}_{k}$; i.e.

$$
\underline{D}_{k}=\underline{G}_{k+1}-\underline{G}_{k}
$$


The adaptive filter weight vector $\underline{H}_{k}$ is composed of the filter weights at time $k$. When the signed regressor algorithm is used for updating the filter weights, $\underline{H}_{k}$ follows the recursion $[2,3]$

$$
\underline{H}_{k+1}=\underline{H}_{k}+\mu\left(a_{k}-\underline{H}_{k}^{T} \underline{X}_{k}\right) \operatorname{sgn}\left(\underline{X}_{k}\right) \quad, k=1,2,3, \ldots
$$

where $\mu$ is the algorithm step size and $\operatorname{sgn}(\cdot)$ is the signum function.

The paper studies the effect of roundoff quantization of $\underline{X}_{k}, a_{k}$, and $\underline{H}_{k}$ on the tracking performance of the algorithm (2). Expressions are derived for the steady state mean square error, the steady state mean square weight deviation, and the values of $\mu$ that minimize each of them.

The paper is organized as follows. Section II presents a mathematical model for the quantized SRA and the assumptions used throughout the analysis. In Section III, the steady state mean square error is derived. The section also derives the step size that minimizes the mean square error and the corresponding minimum mean square error. Section IV is concerned with the derivation of the steady state mean square weight deviation and the corresponding optimum step size. Simulation results are provided in section V. Conclusions of the paper are given in section VI.

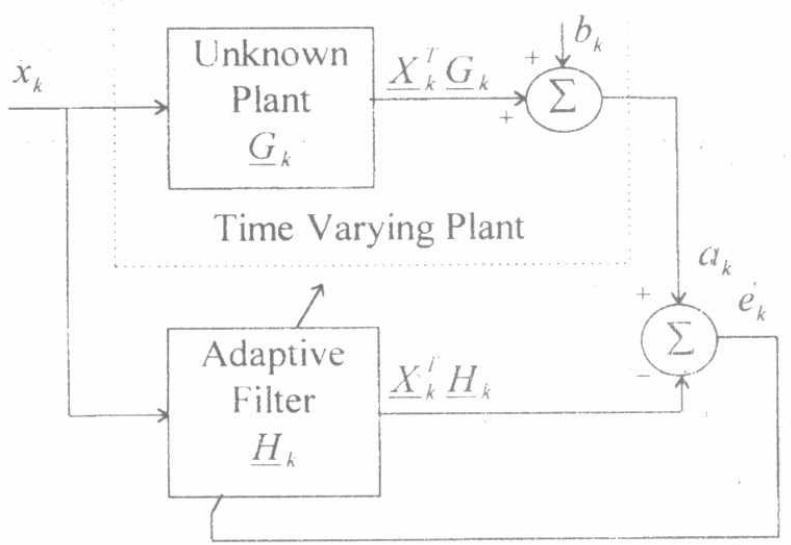

Figure 1. Adaptive Identification of a Time-Varying Plant.

\section{MATHEMATICAL MODEL OF THE QUANTIZED SRA}

The quantized SRA model is shown in figure 2. In this model Q[·] denotes fixed point quantization of [.]. The primed symbols denote quantized values while the unprimed symbols denote the infinite precision values. The input $x_{k}$ is quantized to the wordlength acceptable at the plant input. The same wordlength is used for the adaptive filter input. The plant output is quantized to the wordlength of the adaptive filter output. The difference $e_{k}^{\prime}$ between the quantized plant output and the quantized filter output is used to update the filter weights according to the quantized signed regressor algorithm

$$
\underline{H}_{k+1}^{\prime}=\underline{H}_{k}^{\prime}+Q\left[\mu e_{k}^{\prime} \operatorname{sgn}\left(\underline{X}_{k}^{\prime}\right)\right]
$$

Rounding quantization is assumed. It is assumed also that the saturation thresholds of the quantizers are properly chosen such that the saturation errors are negligible. The weight deviation vector is defined by

$$
\underline{V}_{-k}=\underline{H}_{k}^{\prime}-\underline{G}_{k}
$$




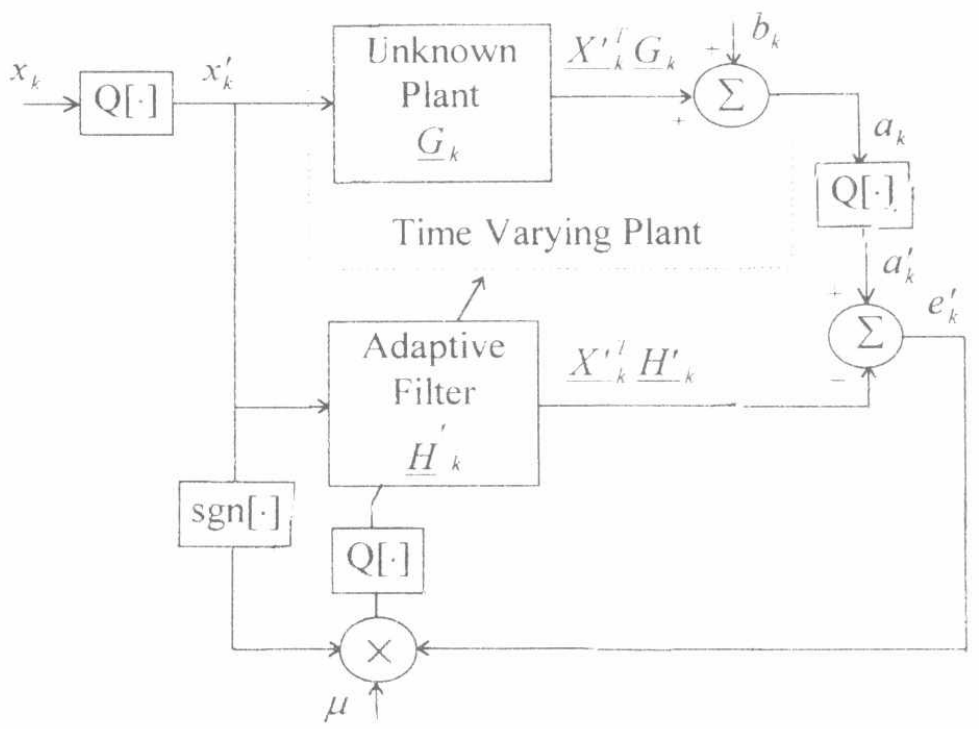

Figure 2. Model of the Quantized SRA

Two performance indices are used to evaluate the tracking capability of the algorithm: the steady state mean square error $\xi=\lim _{k \rightarrow \infty} E\left\{e_{k}^{2}\right\}$ and the steady state mean square weight deviation $\eta=\lim _{k \rightarrow \infty} E\left\{\left\|\underline{V}_{k}\right\|^{2}\right\}$ where $e_{k}$ is the difference between the plant output and the filter output: i.e.

$$
e_{k}=a_{k}-X_{k}^{\prime \prime} H_{k}^{\prime}
$$

and $\left\|\underline{L}_{k}\right\|^{2}=\underline{L}_{k} \underline{L}_{k}$ is the squared norm of $\underline{\underline{r}}_{k}$.

Throughout the analysis, the following assumptions are used Assumption 1: $\underline{X}_{k}$ is zero mean, stationary, Gaussian with a positive definite covariance matrix. Assumption 2: The sequence $\left\{b_{k}\right\}$ is white, stationary, and zero mean with variance $\sigma_{b}^{2}=E\left\{b_{k}^{2}\right\}$.

Assumption 3: $\underline{D}_{k}$ is a stationary sequence of independent zero mean vectors.

Assumption 4: The sequences $\left\{b_{k}\right\},\left\{\underline{V}_{k}\right\},\left\{\underline{X}_{k}\right\}$, and $\left\{\underline{D}_{k}\right\}$ are mutually independent.

Assumption 5: Quantization errors are all zero mean, mutualiy independent, and independent of other signals.

Assumptions 1-4 are typical in the context of nonstationary adaptive filtering [e.g. 2-4] while assumption 5 is typical in the context of finite wordlength effects [e.g. 5-10].

\section{DERIVATION OF THE MEAN SQUARE ERROR}

Using (3), (4), and (1), one obtains

$$
\begin{aligned}
\underline{V}_{k+1} & =\underline{V}_{k}+Q\left[\mu e_{k}^{\prime}\right] \operatorname{sgn}\left(\underline{X}_{k}^{\prime}\right)-\underline{D}_{k} \\
& =\underline{V}_{k}+\left(\mu e_{k}^{\prime}+m_{k}\right) \operatorname{sgn}\left(\underline{X}_{k}^{\prime}\right)-\underline{D}_{k}
\end{aligned}
$$

where $m_{k}$ is the error resulting from quantizing the multiplication of $\mu$ and $e_{k}^{\prime}$ to the wordlength assigned for the filter coefficients. The saturation threshold of the quantizer is denoted by $L$ and 
Proceedings of the $1^{\text {st }}$ ICEENG conference, 24-26 March, 1998.

its number of bits is denoted by $B_{c}$ in addition to:a sign bit. Then, the variance of $m_{k}$ will be given by

$$
\sigma_{c}^{2}=\frac{2^{-2 B_{c}} L_{c}^{2}}{12}
$$

In order that $m_{k}$ may be approximately independent of $e_{k}^{\prime}$, as required by assumption 5 , $\mu \sigma_{h}$ should exceed the least significant bit of the quantization; i.e.

$$
\mu \sigma_{h}>L_{c} 2^{-B_{c}}
$$

From figure 2, The quantized estimation error $e_{k}^{\prime}$ is given by

$$
e_{k}^{\prime}=a_{k}^{\prime}-y_{k}^{\prime}
$$

where $y_{k}^{\prime}$ is the quantized output of the adaptive filter given by

$$
y_{k}^{\prime}=Q\left[{\underline{H^{\prime}}}_{k}^{\prime} \underline{X}_{k}^{\prime}\right]=\underline{H}_{k}^{\prime \prime} \underline{X}_{k}^{\prime}+\gamma_{k}
$$

where $\gamma_{k}$ is the error that results from quantizing ${\underline{H^{\prime}}}_{k}^{\prime} \underline{X}_{-k}^{\prime}$ to $B_{c}$ bits. In practical applications, each one of the $N$ products involved in $\underline{H}_{k}^{\prime \prime} \underline{X}_{k}^{\prime}$ is quantized to, say $B_{y}$ Bits, and the sum is then quantized to $B_{c}$. bits with $B_{v}$ being significantly greater than $B_{c}$. In such a case, $\sigma_{y}^{2}=N \sigma_{v}^{2}+\sigma_{c}^{2}$.

The quantized plant output $a_{k}^{\prime} \cdot$ is given by

$$
a_{k}^{\prime}=a_{k}+\beta_{k}
$$

where $\beta_{k}$ is the quantization error. When the plant output is quantized to $B_{c}$ bits, The variance of $\beta_{k}$ equals $\sigma_{c}^{2}$. From (5), (9) - (11), one obtains

$$
e_{k}^{\prime}=e_{k}+\rho_{k}
$$

where $\rho_{k}=\beta_{k}-\gamma_{k}$ is a zero mean sequence with variance $\sigma_{\rho}^{2}=N \sigma_{y}^{2}+2 \sigma_{c}^{2}$. We shall use the natural assumption that quantization preserves the sign of the signal. Therefore $\operatorname{sgn}\left(\underline{X}_{k}^{\prime}\right)=\operatorname{sgn}\left(\underline{X}_{k}\right)$. Using this fact and substituting (12) in (6), one obtains

$$
\underline{V}_{k+1}=\underline{V}_{k}+\left(\mu\left(e_{k}+\rho_{k}\right)+m_{k}\right) \operatorname{sgn}\left(\underline{X}_{k}\right)-\underline{D}_{k}
$$

From Figure 2, $a_{k}=\underline{X}_{k}^{\prime \prime} \underline{G}_{k}+b_{k}$. Then (4) and (5) imply that

$$
e_{k}=b_{k}-\underline{X}_{k}^{\prime l} \underline{V}_{k}
$$

Substituting (14) in (13), one obtains

$$
\underline{V}_{k+1}=\underline{V}_{k}-\mu \operatorname{sgn}\left(\underline{X}_{k}\right) \underline{X}_{k}^{\prime \prime} \underline{V}_{k}+\left(\mu\left(b_{k}+\rho_{k}\right)+m_{k}\right) \operatorname{sgn}\left(\underline{X}_{k}\right)-\underline{D}_{k}
$$

Squaring (15), taking the expectation of both sides, and then using the assumptions 1-5, one obtains

$$
\begin{aligned}
E\left\{\underline{V}_{k+1} \underline{V}_{k+1}^{\prime}\right\}= & E\left\{\underline{V}_{k} \underline{V}_{k}^{l}\right\}+\mu^{2} E\left\{\operatorname{sgn}\left(\underline{X}_{k}\right) \underline{X}_{k}^{\prime \prime}{ }_{k} \underline{V}_{k} \underline{V}_{k}^{T} \underline{X}_{k}^{\prime} \operatorname{sgn}\left(\underline{X}_{k}^{l}\right)\right\} \\
& -\mu E\left\{\underline{V}_{k} \underline{V}_{k}^{l}\right\} E\left\{\underline{X}_{k}^{\prime} \operatorname{sgn}\left(\underline{X}_{k}^{T}\right)\right\}-\mu E\left\{\operatorname{sgn}\left(\underline{X}_{k}\right) \underline{X}_{k}^{\prime}\right\} E\left\{\underline{V}_{k} \underline{V}_{k}^{T}\right\} \\
& +E\left\{\left(\mu\left(b_{k}+\rho_{k}\right)+m_{k}\right)^{2} \operatorname{sgn}\left(\underline{X}_{k}\right) \operatorname{sgn}\left(\underline{X}_{k}^{T}\right)\right\}+\underline{Q}
\end{aligned}
$$

where $\underline{Q}=E\left\{\underline{D}_{k} \underline{D}_{k}^{T}\right\}$. Assumption 5 implies that $E\left\{\operatorname{sgn}\left(\underline{X}_{k}\right) \underline{X}_{k}^{T}\right\}=E\left\{\operatorname{sgn}\left(\underline{X}_{k}\right) \underline{X}_{k}^{T}\right\}$. Then using [2, (2.9)], one obtains

$$
E\left\{\operatorname{sgn}\left(\underline{X}_{k}\right) \underline{X}_{k}^{\prime \prime}\right\}=\sqrt{\frac{2}{\pi} \frac{R}{\sigma_{x}}}
$$

where $R \Delta E\left\{\underline{X}_{k} \underline{X}_{k}^{T}\right\}$ and $\sigma_{x}^{2}$ is the variance of $x_{k \cdot y}$ Using (17) and the assumptions 2-4, (16) 'becomes 


$$
\begin{aligned}
E\left\{\underline{V}_{k+1} \underline{V}_{k+1}^{T}\right\}= & E\left\{\underline{V}_{k} \underline{V}_{k}^{T}\right\}-\sqrt{\frac{2}{\pi}} \frac{\mu}{\sigma_{x}}\left(\underline{R E}\left\{\underline{V}_{k} \underline{V}_{k}^{T}\right\}+E\left\{\underline{V}_{k} \underline{V}_{k}^{T}\right\} \underline{R}\right) \\
& +\left(\mu^{2}\left(\sigma_{h}^{2}+\sigma_{\rho}^{2}\right)+\sigma_{c}^{2}\right) E\left\{\operatorname{sgn}\left(\underline{X}_{k}\right) \operatorname{sgn}\left(\underline{X}_{k}^{T}\right)\right\} \\
& +\mu^{2} E\left\{\operatorname{sgn}\left(\underline{X}_{k}\right) \underline{X}_{k}^{\prime} \underline{V}_{k} \underline{V}_{k}^{T} \underline{X}_{k}^{\prime} \operatorname{sgn}\left(\underline{X}_{k}^{T}\right)\right\}+\underline{Q}
\end{aligned}
$$

At steady state, $E\left\{\underline{V}_{k+1} \underline{V}_{k+1}^{T}\right\}=E\left\{\underline{V}_{k} \underline{V}_{k}^{T}\right\}$ and then (18) yields

$$
\begin{aligned}
& \left(\mu^{2}\left(\sigma_{b}^{2}+\sigma_{n}^{2}\right)+\sigma_{c}^{2}\right) E\left\{\operatorname{sgn}\left(\underline{X}_{k}\right) \operatorname{sgn}\left(\underline{X}_{k}^{\prime}\right)\right\}+\underline{Q}+\mu^{2} \lim _{k \rightarrow \infty}\left(E\left\{\operatorname{sgn}\left(\underline{X}_{k}\right) \underline{X}_{k}^{\prime \prime} \underline{V}_{k} \underline{V}_{k}^{\prime} \underline{X}_{k}^{\prime} \operatorname{sgn}\left(\underline{X}_{k}^{\prime}\right)\right\}\right)
\end{aligned}
$$

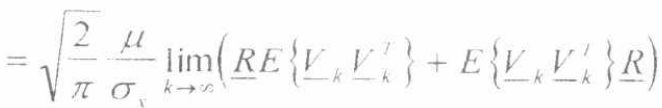

Squaring (14), taking the expectation of both sides, then using the assumptions 1-4, one obtains

$$
\left.\sigma_{e}^{2}(k)=E\left\{e_{k}^{2}\right\}=\sigma_{b}^{2}+E\left\{\underline{X}_{k}^{\prime \prime} \underline{V}_{k} \underline{V}_{k}^{\prime} \underline{X}_{k}^{\prime}\right\} \approx \sigma_{b}^{2}+E \underline{V}_{k}^{\prime} \underline{R}_{k}\right\}
$$

where the last approximation holds under the mild assumption that the mean square input quantization error is small with respect to the minimum eigenvalue of $R$. In the case of white input, this condition implies that

$$
\frac{2^{-2 B_{x}} L_{x}^{2}}{12} \ll<\sigma_{x}^{2}
$$

Where $B_{x}$ and $L_{x}$ are the number of bits and saturation threshold of the input quantizer. For small probability of quantizer saturation, it is sufficient to choose $L_{r}=3 \sigma_{x}$. In such a case (21) will be a very mild condition that may be satisfied with any $B_{x} \geq 3$. Using (20), taking the trace of both sides of (19) and solving for $\sigma_{c}(\infty)$, then the steady state mean square error is given by

$$
\xi=\sigma_{c}^{2}(\infty)=\sigma_{h}^{2}+\frac{\operatorname{tr}(\underline{Q})+N\left(\mu^{2}\left(\sigma_{h}^{2}+\sigma_{p}^{2}\right)+\sigma_{c}^{2}\right)}{\left(\sqrt{8} \mu-\mu^{2} N\right)}
$$

From (22), it is clear that the steady state MSE increases with $\sigma_{c}^{2}$ and $\sigma_{\rho}^{2}$. i.e., the mean square error decreases with increasing the coefficients wordlength $B_{c}$. Equation (22) shows also that the effect of roundoff errors on the mean square error of the SRA is equivalent to an increase of the degree of nonstationarity of the plant and/or the plant noise . The effect of finite wordlength dominates (resp. is dominated by) the effects of the plant nonstationarity and the plant noise when $\operatorname{tr}(Q)+N \mu^{2} \sigma_{b}^{2}$ is smaller (resp. greater ) than $N \sigma_{c}^{2}+N \mu^{2} \sigma_{\rho}^{2}$. The minimum MSE is obtained at $\mu=\mu_{\xi}$ given by

$$
\mu_{\xi}=\frac{\sqrt{2 \pi}}{N \sigma_{r}} v^{2}\left(\sqrt{1+\frac{2}{\pi v^{2}}}-1\right)
$$

where $v$ is defined as

$$
v=\left(\frac{N \sigma_{x}^{2}\left(N \sigma_{c}^{2}+\operatorname{tr}(\underline{Q})\right)}{4\left(\sigma_{b}^{2}+\sigma_{p}^{2}\right)}\right)^{1 / 2}
$$

The corresponding minimum mean square error is

$$
\xi_{\min }=\frac{\pi}{4} N \sigma_{i}^{2}\left(N \sigma_{i}^{2}+\operatorname{tr}(\underline{Q})\right)\left(\sqrt{1+\frac{2}{\pi v^{2}}}+1\right)
$$


Proceedings of the $1^{\text {st }}$ ICEENG conference, 24-26 March, 1998.

\section{MEAN SQUARE WEIGHT DEVIATION}

For small values of $\mu$, the third term on the left hand side of (19) will be small with respect to its right hand side. Then, (19) can be approximated by

$$
\underline{S} \underline{R}+\underline{R} \underline{S}=\sqrt{\frac{\pi}{2}} \frac{\sigma_{x}}{\mu}\left(\underline{Q}+\left(\mu^{2}\left(\sigma_{b}^{2}+\sigma_{\rho}^{2}\right)+\sigma_{c}^{2}\right) \underline{P}\right)
$$

where $\underline{S}=\lim _{k \rightarrow \infty} E\left\{\underline{V}_{k} \underline{V}_{k}^{T}\right\}$ and $\underline{P}=\lim _{k \rightarrow \infty}\left(E\left\{\operatorname{sgn}\left(\underline{X}_{k}\right) \operatorname{sgn}\left(\underline{X}_{k}^{l}\right)\right\}\right)$.

Using (26) with a technique similar to the one given in [4, p.2940], one can show that the steady state mean square weight deviation is given by

$$
\eta=\sqrt{\frac{\pi}{8}} \frac{\sigma_{x}}{\mu}\left(\operatorname{tr}\left(\underline{Q}^{-1}\right)+\left(\mu^{2}\left(\sigma_{b}^{2}+\sigma_{\rho}^{2}\right)+\sigma_{c}^{2}\right) \operatorname{tr}\left(\underline{P}^{-1}\right)\right)
$$

From (27), one deduces that the mean square weight deviation decreases with increasing the coefficient wordlengths. The minimum weight deviation is obtained at $\mu=\mu_{\eta}$ given by

$$
\mu_{\eta}=\sqrt{\frac{\operatorname{tr}\left(\underline{Q} \underline{R}^{-1}\right)}{\left(\sigma_{h}^{2}+\sigma_{\rho}^{2}\right) \operatorname{tr}\left(\underline{P} R^{-1}\right)}+\frac{\sigma_{c}^{2}}{\sigma_{h}^{2}+\sigma_{\rho}^{2}}}
$$

The corresponding minimum mean square weight deviation is

$$
\eta_{\min }=\sqrt{\frac{\pi}{2}} \sigma_{x} \sqrt{\left(\sigma_{h}^{2}+\sigma_{\rho}^{2}\right) \operatorname{tr}\left(\underline{P} \underline{R}^{-1}\right)\left[\operatorname{tr}\left(\underline{Q} \underline{R}^{-1}\right)+\sigma_{c}^{2} \operatorname{tr}\left(\underline{P} \underline{R}^{-1}\right)\right]}
$$

\section{SIMULATION RESULTS}

Simulations are done for the case of adaptive system Identification shown in figure 2. The elements of the increment vector $\underline{D}_{k}$ are zero mean, each of variance $\sigma_{d}^{2}=10^{-6}$. The number of filter taps $N$ is 10. The plant input $x_{k}$ and the plant noise $b_{k}$ are zero mean, Gaussian with variance $\sigma_{r}^{2}=0.09$ and $\sigma_{b}^{2}=10^{-4}$, respectively. The mean square error and mean square weight deviation are calculated by averaging $e_{k}^{2}$ and $\left\|\underline{V}_{k}\right\|^{2}$ respectively over 5000 iterations in the steady state and then averaging over 50 independent runs. The plant input quantizer number of bits $B_{x}$ and saturation threshold $L_{x}$ are chosen to be 8 and 1, respectively. The filter weights saturation threshold $L_{c}$ is 2 . The multiplication $\underline{X}_{k}^{\prime T} \underline{H}_{k}^{\prime}$ is performed with an accumulator with $B_{v}=B_{c}+B_{v}$ bits and then the accumulator output is quantized to $B_{c}$ bits.

Simulation and analytical results of the MSE versus $\mu$ are shown in figure 3. Simulation and analytical results of the MSWD for $\mu=2^{-4}$ are shown in table I. Both figure 3 and table I show a good match between sirnulation and analytical results. This validates the analysis presented in the paper.

\section{CONCLUSIONS}

The effect of finite wordlength on the tracking performance of adaptive filters equipped with the SRA is studied in the context of adaptive plant identification. Expressions of the mean square 


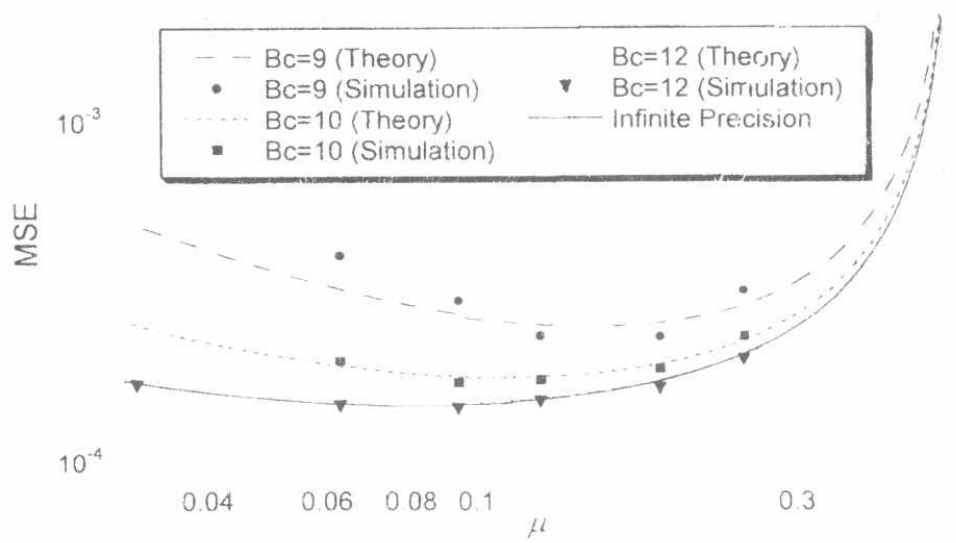

Figure 3. Simulation and analytical results of the MSE

Table I. Simulation and analytical results of the MSWD

\begin{tabular}{|c|c|c|}
\hline Wordlength & MSWD (Theory) & MSWD (Simulation) \\
\hline$B_{c}=9$ & $2.2 \times 10^{-3}$ & $3.36 \times 10^{-3}$ \\
\hline$B_{c}=10$ & $8.93 \times 10^{-4}$ & $1.1 \times 10^{-3}$ \\
\hline$B_{c}=11$ & $5.7 \times 10^{-4}$ & $6.1 \times 10^{-4}$ \\
\hline$B_{c}=12$ & $4.91 \times 10^{-4}$ & $5.2 \times 10^{-4}$ \\
\hline Infinite Precision & $4.65 \times 10^{-4}$ & $4.8 \times 10^{-4}$ \\
\hline
\end{tabular}

error and the mean square weight deviation are derived and validated by compuier simulations. It is found that both the mean square error and the mean square weight deviation decrease ' vith increasing the filter weight wordlengths. The effect of quantization is found to be equivale nt to an increase of the mean square fluctuation of the plant parameters and/or the plant nois'e. It is also found that the effect of input quantization on the algorithm performance is negligibly small for commonly used input wordlengths. A condition at which the effect of plant nonstationarity and plant noise dominate the ef ${ }^{\prime}$ ect of finite wordlength is derived. The derived expressions are in a good agreement with simulation results. These expressions will serve as useful design tools for quantized adaptive filters based on the signed regressor algorithm.

\section{REFERENCES}

[1] B. Widrow. J. M. McCool, M. G. Larimore, C. R. Johnson, " Stationary and nonstationary learning characteristics of the LMS adaptive filter," IEEE Proceedings, Vol. 64, No. 8, Aug. 1976.

[2] E. Eweda, "Analysis and design of a signed regressor LMS algorithm for stationary and nonstationary adaptive filtering with correlated Gaussian data," IEEE Trans. On Circuits\&Systems, vol. 37, No. 11, Nov. 1990.

[3] E. Eweda, "Tracking, analysis of the sign-sign algorithm for nonstationary adaptive filtering with Gaussian data," IEEE Trans. on Signal Processing, vol. 45, No. 5, May. 1997.

[4] E. Eweda, "Comparison of RLS, LMS, and Sign Algorithms for Tracking Randomly TimeVarying Channels," IEEE Trans. On Signal Processing, vol. 42, No. 1.1, Nov. 1994.

[5] E. Eweda, N. Yousef, and Salwa El-Ramly, "Effect of finite wordlength on the performance of an adaptive filter equipped with the signed regressor algorithm," Proceedings of the IEEE Global Telecommunications Conference, London, UK, vol. 2, pp. 1325-1329, November 1996. 
[6] J. C. M. Bermudez, N. J. Bershad, "Transient and Tracking performance analysis of the quantized LMS algorithm for time-varying system identification," IEEE Trans. on Signal Processing, vol. 44, No. 8, pp. 1990-1997, Aug. 1996.

[7] E. Eweda, N. Yousef, and Salwa El-Ramly, "Effect roundoff errors on adaptive digital signal processing using the sign algorithm," Proceedings of the International Conference on Signal Processing Applications and Technology, Boston, USA, vol.1, pp. 38-42, Oct. 1996.

[8] R.D. Gitlin et al. , "On the design of gradient algorithms for digitally implemented adaptive filters." IEEE Trans. Circuit theory, vol. CT-20, p.p. 125-136, March 1973.

[9] J.M. Cioffi, "Limited-Precision effects in adaptive filtering," IEEE Trans. Circuits and Systems, vol. CAS-32, no. 1, February 1984.

[10]C. Caraiscos and B. Liu, "A roundoff error analysis of the LMS adaptive algorithm," IEEE Trans. Acoustics, Speech and signal processing, vol. ASSP-32, no. 1, February 1984.

III] S. Haykin, Adaptive filter theory, Third edition, Prentice-Hall Int, 1996. 
